\title{
AN ALTERNATIVE SEMANTICS FOR MODAL PREDICATE-LOGIC
}

(A) In this paper I present a realistic semantics for first-order modal predicate-logic that differs in two respects from the standard TarskiKripke approach.

Firstly, the background theory is not axiomatic set theory supplemented by the concept possible world, but a theory of basic intensional entities: propositions, properties and relations; ${ }^{1}$ this theory will here be developed only as far as it is necessary for the semantics of an elementary language, that is, the only properties and relations considered are properties of, and relations between individuals. In standard (extensional) ontology intensional entities are reduced to sets and possible worlds (and individuals; they are complex sets involving in their specification possible worlds and/or individuals) ${ }^{2}$ in intensional ontology, however, sets (of individuals) are reduced to properties (of individuals), and possible worlds to propositions. The latter reduction is more natural than the former, and the inveterate skepticism against intensional entities as basic ("What, after all, are these things?? When are they identical??") can be dispelled. For intensional ontology is completely on a par with set theory. All we really know about sets is stated in the axioms of set theory; all we really know about properties, relations and propositions is stated in the axioms of intensional ontology; the latter axioms are no less precise than the former, and identity conditions for intensional entities are not left unclear.

Secondly, the basic semantic concept is different; expressions do not have extensions relative to possible worlds in some interpretation, but absolutely intend intensions. Sentences intend propositions, monadic predicates intend properties, polyadic predicates intend relations, individual-constants intend individuals. To intend is not to mean; we can, however, think of the intension of an expression as an approximation to its meaning, such that expressions that mean the same always intend the same, but not vice versa (while expressions that intend the same have the same extension, but not vice versa). ${ }^{3}$ The central act (so to speak) of my semantics is not the stating of a definition of the concept being an interpretation of language $L$, but rather the stating of semantic

(C) 1992 Kluwer Academic Publishers. Printed in the Netherlands. 
axioms that show how the intensions of more complex expressions of $L$ are determined by the intensions of simpler expressions of $L$ that are their parts. (All of the axioms having to do with logical operators are equations; by repeated application of the axioms (the intension of) each sentence of $L$ can be completely analyzed.) Of course, the definition of logical truth will look rather different on this approach. (In fact, four semantic concepts of truth will be defined for $L$ : truth, simpliciter and relative, ontological truth and logical truth.)

The aim of this paper is not to point out problems that the standard approach to modal semantics cannot deal with, while the non-standard one can; it is rather to show (especially in the semantical part) that the same problems the standard approach successfully deals with can be solved in a rather different manner, which, on the whole, seems to me to be more satisfactory intuitively and to be more in line with philosophical tradition than the usual way. But even if it should seem otherwise to the reader, the gain of knowledge obtained by going a different route to the same summit ought not to be despised.

(B) I begin by stating the background theory of basic intensional entities. Like set theory it is a first-order theory. All quantifiable variables are of one kind; they can replace any singular term, and no other expression.

\section{Expressions Specific to the Theory}

$Z^{0}(\tau)$ means $\tau$ is an individual;

$Z^{1}(\tau)$ means $\tau$ is a proposition;

$Z^{(0\rangle}(\tau)$ means $\tau$ is a property (of individuals);

$Z^{\langle 0,0\rangle}(\tau)$ means $\tau$ is a dyadic relation (between individuals);

$Z^{\langle 0,0,0\rangle}(\tau)$ means $\tau$ is a triadic relation (between individuals);

$\left(\tau P \tau^{\prime}\right)$ means proposition $\tau$ is (intensional) part of proposition $\tau^{\prime}$; $\left(\tau, \tau_{1}, \ldots, \tau_{n}\right)$ means the proposition which is the saturation of $\tau$ by $\tau_{1}, \ldots, \tau_{n}$ (equivalently the proposition which is the concatenation of $\tau$ with $\left.\tau_{1}, \ldots, \tau_{n}\right)$

$\lambda o \pi[o]$ means the property which results from $\pi[\tau]$ by the extraction of $\tau(\pi[\tau]$ is a singular term; $\tau$ not in $\pi[o])$;

$\lambda o_{1}, \ldots o_{n} \pi\left[o_{1}, \ldots, o_{n}\right](2 \leqslant n)$ means the $n$-adic relation which results 
from $\pi\left[\tau_{1}, \ldots, \tau_{n}\right]$ by the extraction of $\tau_{1}, \ldots, \tau_{n}\left(\pi\left[\tau_{1}, \ldots, \tau_{n}\right]\right.$ is a singular term; $o_{1}, \ldots, o_{n}$ are different from each other; $\tau_{1}, \ldots, \tau_{n}$ are different from each other and not in $\left.\pi\left[o_{1}, \ldots, o_{n}\right]\right)$.

$o, o^{\prime}, o^{\prime \prime} \ldots$ are nonquantifiable variables that occur only within the scope of an occurrence of $\lambda$; they can replace any singular term, and no other expression; they are used instead of normal variables for no other reason than better readability $\left(\pi\left[\tau_{1}, \ldots, \tau_{n}\right]\right.$ may already contain lots of normal variables).

The convention governing the use of square brackets in stating axiomor definition-schemata is as follows: $\pi\left[\tau_{1}, \ldots, \tau_{n}\right]$ implies that $\tau_{1}$ occurs (syntactically correctly) in $\pi$ at some place(s) intended by the square bracketing (and marked by the numeral "1"), that $\tau_{2}$ occurs in $\pi$ at some other place(s) intended by the square bracketing (and marked by the numeral " 2 "), . . If $\tau_{r}$ is a variable, its occurrence is supposed to be free at each place intended by the square bracketing.

$w$ means the (real) world.

\section{Logical Expressions}

non means not;

a. means and;

$o$. means or;

imp. means implies;

equ. means equivalent.

Binding strength diminishes from top to bottom, and brackets may be saved accordingly; moreover, brackets are omitted as exemplified in $A$ a. B a. C, $A$ o. B o. C;

$\vee \nu$ means there is a such that;

$\wedge \nu$ means for all $\nu$;

$\iota \nu$ means the $\nu$ such that;

$\left(\tau=\tau^{\prime}\right)$ means $\tau$ is identical with $\tau^{\prime}$.

The brackets around $\tau P \tau^{\prime}$ and $\tau=\tau^{\prime}$ are not written, except they form the scope of a quantifier $(\wedge, \vee, \iota)$. 


\section{The Logic}

Standard classical first-order predicate-logic with identity and definite description (just as for most systems of set theory). Theorems and axioms with free variables are equivalent to their universal closure.

\section{The First Two Groups of Axioms}

(a) The first group of axioms characterizes the predicates $Z^{1}(\tau)$ and $\tau P \tau^{\prime}$ (axiom-schemata are considered as axioms):

AXIOM 0. $\wedge x \wedge y\left(x P y\right.$ imp. $\left.Z^{1}(x) a . Z^{1}(y)\right)$; Axiom 0 simply makes explicit what has been said about the meaning of $\tau P \tau^{\prime}$.

AXIOM 1. $\wedge x \wedge y \wedge z(x P y$ a. $y P z$ imp. $x P z)$; the transitivity of the part-predicate.

AXIOM 2. $\wedge x\left(Z^{1}(x)\right.$ imp. $\left.x P x\right)$; "every proposition is part of itself".

AXIOM 3. $\wedge x \wedge y(x P y$ a. $y P x$ imp. $x=y)$; "propositions which are part of each other are identical".

AXIOM 4. $\vee z\left[Z^{1}(z) a . \wedge y\left(Z^{1}(y) a . A[y]\right.\right.$ imp. $\left.y P z\right) a . \wedge x\left(Z^{1}(x) a\right.$. $\wedge y\left(Z^{1}(y)\right.$ a. $\left.A[y] \mathrm{imp} . y P x\right)$ imp. $\left.\left.z P x\right)\right]$; "there is a proposition such that every $A$-proposition is part of it, and which is part of every proposition that every $A$-proposition is part of ", "there is a smallest proposition every $A$-proposition is part of". (Variables in axiom- and definition-schemata are representative for the (syntactically) "right" variables, and are supposed to occur only in the places indicated.)

For stating Axiom 5 in a fairly convenient manner I need two definitions:

DEFINITION 1. $c x A[x]:=\imath z\left[Z^{1}(z) a . \wedge y\left(Z^{1}(y) a . A[y]\right.\right.$ imp. $\left.y P z\right)$ a. $\wedge x\left(Z^{1}(x)\right.$ a. $\wedge y\left(Z^{1}(y)\right.$ a. $A[y]$ imp. $\left.y P x\right)$ imp. $\left.\left.z P x\right)\right]$;

"the proposition which is the conjunction of all $A$-propositions is the smallest proposition every $A$-proposition is part of". Existence and uniqueness are guaranteed for the definiens by Axioms 3 and 4. 
DEFINITION 2. $M i(\tau):=Z^{1}(\tau)$ a. $\wedge y\left(Z^{1}(y)\right.$ imp. $\left.\tau P y\right)$; “ $\tau$ is an absolutely minimal proposition iff $\tau$ is a proposition which is part of every proposition".

AXIOM 5. $\wedge y[y P c x A[x] a$. non $M i(y)$ imp. $\vee z(z P y a$. non $M i(z) a$. $\vee x(z P x a . A[x]))]$. " $\tau P \tau$ ' $a$. non $M i(\tau)$ " will always be read as "proposition $\tau$ is a non-trivial part of proposition $\tau^{\prime \prime}$; then one can read Axiom 5 as "every proposition which is a non-trivial part of the proposition which is the conjunction of all $A$-propositions, has a proposition as non-trivial part that is also part of an $A$-proposition"; after the comma I can also read "non-trivially overlaps an $A$-proposition".

Axioms 0-5 state - as far as this is possible in a language that has no means for quantifying over properties of propositions - that the totality of propositions has the structure of a complete Boolean algebra. ${ }^{4}$

For stating Axiom 6 in a convenient manner I need another definition:

DEFINITION 3. $A t(\tau):=Z^{1}(\tau)$ a. $\wedge y(y P \tau$ a. non $M i(y)$ imp. $y=$ $\tau)$; " $\tau$ is an atomic proposition iff $\tau$ is a proposition such that every proposition which is a non-trivial part of it, is identical to it".

AXIOM 6. $\wedge x \wedge y\left(Z^{1}(x) a . Z^{1}(y) a . \wedge z(A t(z) a . z P x\right.$ imp. $z P y)$ imp. $x P y)$; "if every atomic proposition which is part of a proposition $x$ is also part of a proposition $y$, then $x$ is part of $y$ ".

Axioms 0-6 state that the totality of propositions has the structure of a complete and atomistic Boolean algebra. The selection of Axioms $0-6$ from the many alternative formulations of a complete and atomistic Boolean algebra was primarily determined by the intuitive perspicacity of that system, and secondarily by its handiness in proving theorems. (Presumably there is a more perspicuous formulation of Axiom 5, which the reader is invited to find.)

(b) The second group of axioms characterizes the constant $w$. I shall state four definitions before going ahead:

DEFINITION 4. $t:=c x$ non $x=x$; "the tautological proposition is the proposition which is the conjunction of all propositions which are not self-identical"; this reading will be justified below. 
DEFINITION 5. $c:=c x(x=x)$; "the contradictory proposition is the proposition which is the conjunction of all propositions"; this reading will be justified below.

DEFINITION 6. $M a(\tau):=Z^{1}(\tau) a . \wedge y\left(Z^{1}(y)\right.$ imp. $\left.y P \tau\right)$; " $\tau$ is an absolutely maximal proposition iff $\tau$ is a proposition every proposition is part of".

DEFINITION 7. $T o(\tau):=Z^{1}(\tau) a . \wedge y(\tau P y a$. non $M a(y)$ imp. $y=$ $\tau)$; " $\tau$ is a total proposition iff $\tau$ is a proposition such that every proposition it is non-trivially part of, is identical with it".

Definitions 4 and 5 are complementary to each other, and so are Definitions 2 and 6, and Definitions 3 and 7.

AXIOM 7. Non $w=t$; "the world is not the tautological proposition".

AXIOM 8. Non $w=c$; "the world is not the contradictory proposition".

AXIOM 9. To $(w)$; "the world is a total proposition".

5. Some Theorems Derivable from the First Group of Axioms

Firstly:

DEFINITION 8. $\tau^{\prime} \rightarrow \tau:=\tau P \tau^{\prime}$; “ $\tau^{\prime}$ entails $\tau$ iff proposition $\tau$ is part of proposition $\tau^{\prime \prime}$. Then it is easy to prove:

THEOREM 1. $\wedge x\left(Z^{1}(x) a . \wedge y\left(Z^{1}(y)\right.\right.$ imp. $\left.y \rightarrow x\right)$ equ. $\left.x=t\right)$; " the tautological proposition is the only proposition that is entailed by every proposition"; this justifies the reading of Definition 4.

THEOREM 2. $\wedge x\left(Z^{1}(x) a . \wedge y\left(Z^{1}(y)\right.\right.$ imp. $\left.x \rightarrow y\right)$ equ. $\left.x=c\right)$; " the contradictory proposition is the only proposition that entails every proposition"; this justifies the reading of Definition 5. 
Secondly:

DEFINITION 9. $\left(\tau \wedge \tau^{\prime}\right):=c x\left(x P \tau o . x P \tau^{\prime}\right)$; "the proposition which is the conjunction of $\tau$ and $\tau^{\prime}$ is the proposition that is the conjunction of every proposition which is part of $\tau$ or part of $\tau^{\prime \prime}$.

DEFINITION 10. $\left(\tau \vee \tau^{\prime}\right):=c x\left(x P \tau a . x P \tau^{\prime}\right)$; "the proposition which is the disjunction of $\tau$ and $\tau^{\prime}$ is the proposition that is the conjunction of every proposition which is both part of $\tau$ and part of $\tau^{\prime \prime}$.'

DEFINITION 11. $\neg \tau:=c x(A t(x) a$. non $x P \tau)$; "the proposition which is the negation of $\tau$ is the conjunction of every atomic proposition that is not a part of $\tau$ '.

Then it is easy to prove (for example):

THEOREM 3. $\wedge x \wedge y\left(Z^{1}(x)\right.$ a. $Z^{1}(y)$ imp. $\wedge z(z \rightarrow(x \wedge y)$ equ. $z \rightarrow x \quad a . z \rightarrow y)$ ).

THEOREM 4. $\wedge x \wedge y\left(Z^{1}(x)\right.$ a. $Z^{1}(y)$ imp. $\wedge z((x \vee y) \rightarrow z$ equ. $x \rightarrow z$ a. $y \rightarrow z)$ ).

THEOREM 5. $\wedge x\left[Z^{1}(x)\right.$ imp. $\wedge y(x \rightarrow y a . \neg x \rightarrow y$ equ. $y=t) a$. $\wedge y(y \rightarrow x a . y \rightarrow \neg x$ equ. $y=c)$ ]. (From now on I leave the intuitive readings of theorems in most cases to the reader.)

Thirdly:

DEFINITION 12. $d x A[x]:=c x \wedge y\left(Z^{1}(y) a . A[y]\right.$ imp. $\left.x P y\right)$; "the proposition which is the disjunction of all $A$-propositions is the proposition which is the conjunction of all propositions which are part of every $A$-proposition". Then it can be proved:

THEOREM 6. $d x A[x]=\neg c x \vee y\left(Z^{1}(y) a . A[y] a . x=\neg y\right)$.

Fourthly:

DEFINITION 13. Con $(\tau):=Z^{1}(\tau)$ a. $\wedge y\left(Z^{1}(y)\right.$ imp. non $\tau \rightarrow y o$. non $\tau \rightarrow \neg y$ ); " $\tau$ is a consistent proposition iff $\tau$ is a proposition, and 
there is no proposition such that $\tau$ entails both it and the proposition that is its negation".

DEFINITION 14. $\operatorname{Max}(\tau):=Z^{1}(\tau)$ a. $\wedge y\left(Z^{1}(y)\right.$ imp. $\tau \rightarrow y \quad o$. $\tau \rightarrow \neg y$ ); " $\tau$ is a maximal proposition iff $\tau$ is a proposition, and there is no proposition such that $\tau$ entails neither it nor the proposition that is its negation".

Then it can be proved:

THEOREM 7. $\wedge y\left[Z^{1}(y)\right.$ imp. (non $y=c$ equ. Con $\left.\left.(y)\right)\right]$.

THEOREM 8. $\wedge y(T o(y)$ equ. $\operatorname{Max}(y))$.

6. Some Theorems Derivable From the First and Second Group of Axioms

Firstly:

DEFINITION 15. $M C(\tau):=\operatorname{Max}(\tau) a$. Con $(\tau)$; " $\pi$ is a maximal-consistent proposition iff $\tau$ is a maximal and consistent proposition". By Theorems 7 and 8, Axioms 8 and 9 it follows immediately:

THEOREM 9. $M C(w)$.

Since the (real) world is a maximal-consistent proposition, the possible worlds are the maximal-consistent propositions; hence we may read $\mathrm{MC}(\tau)$ also as " $\tau$ is a possible world". 5

Secondly:

DEFINITION 16. (a) $T(\tau):=w \rightarrow \tau$

(b) $F(\tau):=w \rightarrow \neg \tau$; “ $\tau$ is true (false) iff $\tau(\neg \tau)$ is entailed by the world".

DEFINITION 17. (a) $T\left(\tau, \tau^{\prime}\right):=M C\left(\tau^{\prime}\right) a . \tau^{\prime} \rightarrow \tau$

(b) $F\left(\tau, \tau^{\prime}\right):=M C\left(\tau^{\prime}\right)$ a. $\tau^{\prime} \rightarrow \neg \tau$; “ $\tau$ is true (false) in $\tau^{\prime}$ iff $\tau^{\prime}$ is a possible world that entails $\tau(\neg \tau)$ ".

DEFINITION 16 defines the ontological concepts of truth and falsity 
(which apply to propositions, while the semantic concepts of truth and falsity apply to sentences); Definition 17 is its generalization.

Various truth-laws can be derived. For example:

THEOREM 10. $\wedge y\left[Z^{1}(y) \mathrm{imp}\right.$. $(T(y)$ equ. non $\left.F(y))\right]$; "ontological bivalence".

THEOREM 11. $\wedge x \wedge y\left[Z^{1}(x) a . Z^{1}(y)\right.$ imp. $(T((x \vee y))$ equ. $T(x) o$. $T(y))]$.

THEOREM 12. $\wedge x \wedge y\left[Z^{1}(x)\right.$ a. $M C(y)$ imp. $(T(\neg x, y)$ equ. $F(x, y))]$.

Thirdly:

THEOREM 13. $\wedge x\left[Z^{1}(x)\right.$ imp. (non $x \rightarrow c$ equ. $\left.\left.\vee y T(x, y)\right)\right]$. Theorem 13 states the equivalence of two traditional conceptions of propositional possibility. I choose the more recent one for a definiens:

DEFINITION 18. $P(\tau):=\bigvee y T(\tau, y)$; " $\tau$ is a (ontologically) possible proposition iff $\tau$ is true in some possible world". 6

Using Axioms 7 and 8 it is easy to prove

THEOREM 14. $\vee y(P(y)$ a. $P(\neg y))$. Theorem 14 is important in showing that (ontological) truth, possibility and necessity (non $P(\neg \tau)$ ) do not collapse into each other.

Fourthly: Let $R_{1}, R_{2}, \ldots, R_{k}, \ldots$ be two-place predicates such that

(R) $\wedge x \wedge y\left(x R_{k} y\right.$ imp. $M C(x)$ a. $\left.M C(y)\right)$; these predicates can be used to define relativized possibility-predicates:

DEFINITION 19. $P_{k}\left(\tau, \tau^{\prime}\right):=\bigvee y\left(\tau^{\prime} R_{k} y a . T(\tau, y)\right)$; “ $\tau$ is $k$-possible in $\tau^{\prime}$ iff there is some world $k$-accessible from $\tau^{\prime}$ such that $\tau$ is true in it". (If we want to have $\wedge x \wedge y\left(T(x, y)\right.$ imp. $\left.P_{k}(x, y)\right)$, we must have $\wedge x\left(M C(x)\right.$ imp. $\left.x R_{k} x\right)$.) They can also be used to define possibilityfunctors: 
DEFINITION 20. $p_{k}(\tau):=d x \vee y\left(x R_{k} y a . T(\tau, y)\right)$; " the proposition that is the $k$-possibility of $\tau$ is the proposition that is the disjunction of every possible world from which some world is $k$-accessible in which $\tau$ is true"; equivalently: ". . . is the proposition that is the disjunction of every possible world in which $\tau$ is $k$-possible". It can be proved:

THEOREM 15. $\wedge x \wedge y\left(T\left(p_{k}(x), y\right)\right.$ equ. $\left.P_{k}(x, y)\right)$, using beside (R) and the definitions the theorems,

THEOREM 16. $\wedge y[M C(y)$ a. $y \rightarrow d z A[z]$ imp. $\vee z(A[z] a . y \rightarrow z)]$,

THEOREM 17. $\wedge z \wedge y(M C(y) a . M C(z) a \cdot y \rightarrow z$ imp. $z=y)$,

THEOREM 18. $\wedge y\left(Z^{1}(y) a . A[y]\right.$ imp. $\left.y \rightarrow d z A[z]\right)$.

7. The Third Group of Axioms, and Theorems Derivable by Their Help

The third group of axioms characterizes the categorial predicates $Z^{n}(\tau)$ and the saturation-operator (or rather -operators) $\left(\tau, \tau_{1}, \ldots, \tau_{n}\right)$.

AXIOM 10. $\wedge x\left(Z^{n}(x)\right.$ imp. non $\left.Z^{m}(x)\right)$, where $n$ and $m$ are different types out of: $0,1,\langle 0\rangle,\langle 0,0\rangle, \ldots$; the exclusiveness of ontological categories.

AXIOM 11. $\vee x Z^{0}(x)$; "there are individuals". No other existencepostulate of the form $\vee x Z^{n}(x)$ is necessary.

AXIOM 12. $\wedge x_{1} \ldots \wedge x_{n} \wedge y\left[Z^{\langle n\rangle}(y) a . Z^{0}\left(x_{1}\right) a . \ldots a . Z^{0}\left(x_{n}\right)\right.$ imp. $\left.Z^{1}\left(\left(y, x_{1}, \ldots, x_{n}\right)\right)\right]$; "the saturation of a $n$-place relation (between individuals) by $n$ ordered individuals is a proposition". Here properties are considered to be 1-place relations; subscript $\langle n\rangle$ is short for a sequence of $\boldsymbol{n}$ tokens of 0 , separated by commas and included in brackets \langle\rangle$;\langle 3\rangle$, for example, is short for $\langle 0,0,0\rangle$.

AXIOM 13. $\wedge x_{1} \ldots \wedge x_{n} \wedge y$ (non $Z^{\langle n\rangle}(y) o$. non $Z^{0}\left(x_{1}\right) o . \ldots o$. non $Z^{0}\left(x_{n}\right)$ imp. $\left.\left(y, x_{1}, \ldots, x_{n}\right)=c\right)$; "if an entity is not an $n$-place relation or some of the ordered $n$ entities it is saturated by is not an individual, then the saturation of it by the $n$ ordered entities is the contradictory proposition". 
From Axioms 12 and 13 we get immediately

THEOREM 19. $\wedge y \wedge x_{1} \ldots \wedge x_{n} Z^{1}\left(\left(y, x_{1}, \ldots, x_{n}\right)\right)$; that is, Axiom 12 and Axiom 13 justify the reading of $\left(\tau, \tau_{1}, \ldots \tau_{n}\right)$ as "the proposition which is the saturation of $\tau$ by $\tau_{1}, \ldots, \tau_{n}$ "; Axiom 12 provides for any proper case of saturation, Axiom 13 for any improper one.

The predicate of satisfaction (by individuals, or rather the predicates of satisfaction) is (are) defined as follows:

DEFINITION 21. $\tau\left(\tau_{1}, \ldots, \tau_{n}\right):=T\left(\left(\tau, \tau_{1}, \ldots, \tau_{n}\right)\right)$; “ $\tau_{1}, \ldots, \tau_{n}$ satisfy $\tau$ ( $\tau$ applies to $\tau_{1}, \ldots, \tau_{n} ; \tau_{1}, \ldots, \tau_{n}$ exemplify $\tau$ ) iff the proposition which is the saturation of $\tau$ by $\tau_{1}, \ldots, \tau_{n}$ is true". Then it is easy to prove (using Axioms 13 and 8):

THEOREM 20. $\wedge y \wedge x_{1} \ldots \wedge x_{n}\left(y\left(x_{1}, \ldots, x_{n}\right)\right.$ imp. $Z^{\langle n\rangle}(y) a$. $\left.Z^{0}\left(x_{1}\right) a . \ldots a . Z^{0}\left(x_{n}\right)\right)$. Using Axiom 10, I get from Theorem 20, for example,

THEOREM 21. $\wedge y\left(Z^{\langle 0\rangle}(y)\right.$ imp. non $\left.y(y)\right)$; " no property applies to itself".

Definition 21 can be generalized:

DEFINITION 22. $\left(\tau\right.$ in $\left.\tau^{\prime}\right)\left(\tau_{1}, \ldots, \tau_{n}\right):=T\left(\left(\tau, \tau_{1}, \ldots, \tau_{n}\right), \tau^{\prime}\right)$; " $\tau_{1}, \ldots, \tau_{n}$ satisfy $\tau$ in $\tau^{\prime}$ iff the proposition which is the saturation of $\tau$ by $\tau_{1}, \ldots, \tau_{n}$ is true in $\tau^{\prime \prime \prime}$.

The next axiom states the sufficient identity condition for properties and relations:

AXIOM 14. $\wedge x \wedge y\left(Z^{\langle n\rangle}(x) a . Z^{\langle n\rangle}(y) a\right.$. $\wedge z_{1} \ldots \wedge z_{n}\left(\left(x, z_{1}, \ldots, z_{n}\right)=\left(y, z_{1}, \ldots, z_{n}\right)\right)$ imp. $\left.x=y\right)$.

In the axiom-system so far developed it is possible to prove (and to link) two principles well-known from orthodox intensional semantics. Without Axiom 14 it is provable:

THEOREM 22. $\wedge x \wedge y\left(Z^{1}(x) a . Z^{1}(y) a . \wedge z(T(x, z)\right.$ equ. $T(y, z))$ 
imp. $x=y$ ); "propositions are identical, if they are true in the same worlds". From Theorem 22 it follows by Axiom 14:

THEOREM 23. $\wedge x \wedge y\left(Z^{\langle n\rangle}(x) a . Z^{\langle n\rangle}(y) a . \wedge u \wedge z_{1} \ldots \wedge z_{n}((x\right.$ in $u)\left(z_{1}, \ldots, z_{n}\right)$ equ. $(y$ in $\left.u)\left(z_{1}, \ldots, z_{n}\right)\right)$ imp. $\left.x=y\right)$; " $n$-place relations are identical if they apply to the same $n$ ordered individuals in each world".

Theorems 22 and 23 show that propositions and $n$-place relations can only serve as intensions for sentences and $n$-place predicates, not as meanings; meanings need to be finer differentiated, what becomes apparent when we consider epistemic contexts. That meanings are not dealt with in this paper should not be taken as the tacit admission that they cannot be represented in a properly extended intensional background theory. If we move up the type-hierarchy to relations between properties/relations (of individuals) and individuals, then we can reconstruct, for example, structured propositions as ordered sequences starting with a property or relation (of individuals) and ending with a tail of individuals (compare the treatment of ordered sequences of individuals only in section C,3 of this paper).

Finally I offer an important definition that gives the ontological correlates of restricted all-quantifiers:

DEFINITION 23. $a^{\tau^{\prime}}(\tau):=c x \vee z\left(\tau^{\prime}(z) a .(\tau, z) \rightarrow x\right)$; "the $\tau^{\prime}$-relative all-proposition of $\tau$ is the proposition which is the conjunction of every proposition entailed by some proposition resulting when $\tau$ is saturated by an individual satisfying $\tau^{\prime \prime}$. It can be proved:

THEOREM 24. $\wedge x \wedge y\left[T\left(a^{y}(x)\right)\right.$ equ. $\wedge z(y(z)$ imp. $\left.x(z))\right]$.

8. The Fourth Group of Axioms, and Theorems Derivable by Their Help

The fourth group of axioms characterizes the extraction-operator $\lambda$ :

AXIOM 15. $\wedge x_{1} \ldots \wedge x_{n}\left[Z^{0}\left(x_{1}\right) a . \ldots a . Z^{0}\left(x_{n}\right) a\right.$.

$Z^{1}\left(\pi\left[x_{1}, \ldots, x_{n}\right]\right)$ imp. $\left(\lambda o_{1} \ldots o_{n} \pi\left[o_{1}, \ldots, o_{n}\right]\right.$,

$\left.\left.x_{1}, \ldots, x_{n}\right)=\pi\left[x_{1}, \ldots, x_{n}\right]\right]$; "the $n$-place relation which results from the proposition $\pi\left[x_{1}, \ldots, x_{n}\right]$ by the extraction of individuals 
$x_{1}, \ldots, x_{n}$ yields a proposition that is identical with $\pi\left[x_{1}, \ldots, x_{n}\right]$ if it is saturated by $x_{1}, \ldots, x_{n} "$.

AXIOM 16. $\wedge x_{1} \ldots \wedge x_{n}$ (non $Z^{1}\left(\pi\left[x_{1}, \ldots, x_{n}\right]\right)$ imp.

$\left.\left(\lambda o_{1} \ldots o_{n} \pi\left[o_{1}, \ldots, o_{n}\right], x_{1}, \ldots, x_{n}\right)=c\right)$; "the $n$-place relation which results from the non-proposition $\pi\left[x_{1}, \ldots, x_{n}\right]$ by the extraction of entities $x_{1}, \ldots, x_{n}$ yields the contradictory proposition if it is saturated by $x_{1}, \ldots, x_{n}$ ". Axiom 15 takes care of any proper case of extraction, Axiom 16 (together with Axiom 13) of any improper one.

By Axioms 15, 16 and 13:

THEOREM 25. $\wedge x_{1} \ldots \wedge x_{n}\left[\left(\lambda o_{1} \ldots o_{n} \pi\left[o_{1}, \ldots, o_{n}\right]\right.\right.$, $\left.x_{1}, \ldots, x_{n}\right)=\pi\left[x_{1}, \ldots, x_{n}\right] o .\left(\lambda o_{1} \ldots o_{n} \pi\left[o_{1}, \ldots, o_{n}\right]\right.$, $\left.\left.x_{1}, \ldots, x_{n}\right)=c\right]$.

By Axiom 15 and 13:

THEOREM 26. $\vee x_{1} \ldots \vee x_{n}\left(Z^{0}\left(x_{1}\right) a . \ldots a . Z^{0}\left(x_{n}\right) a\right.$. $Z^{1}\left(\pi\left[x_{1}, \ldots, x_{n}\right]\right) a$. non $\left.\pi\left[x_{1}, \ldots, x_{n}\right]=c\right)$ imp.

$Z^{\langle n\rangle}\left(\lambda o_{1} \ldots o_{n} \pi\left[o_{1}, \ldots, o_{n}\right]\right)$.

Axiom 17 is designed to provide for the case left open by Theorem 26:

AXIOM 17. non $\vee x_{1} \ldots \vee x_{n}\left(Z^{0}\left(x_{1}\right) a \ldots a\right.$. $Z^{0}\left(x_{n}\right)$ a. $Z^{1}\left(\pi\left[x_{1}, \ldots, x_{n}\right]\right)$ a. non $\left.\pi\left[x_{1}, \ldots, x_{n}\right]=c\right)$ imp. $Z^{\langle n\rangle}\left(\lambda o_{1} \ldots o_{n} \pi\left[o_{1}, \ldots, o_{n}\right]\right)$.

By Theorem 26 and Axiom 17:

THEOREM 27. $Z^{\langle n\rangle}\left(\lambda o_{1} \ldots o_{n} \pi\left[o_{1}, \ldots, o_{n}\right]\right)$; Theorem 27 justifies the reading of $\lambda o_{1} \ldots o_{n} \pi\left[o_{1}, \ldots, o_{n}\right]$ as "the n-place relation resulting....".

By Axioms 15, 16 and 13:

THEOREM 28. Non $\bigvee x_{1} \ldots \vee x_{n}\left(Z^{0}\left(x_{1}\right) a . \ldots a . Z^{0}\left(x_{n}\right) a\right.$. $Z^{1}\left(\pi\left[x_{1}, \ldots, x_{n}\right]\right) a$. non $\left.\pi\left[x_{1}, \ldots, x_{n}\right]=c\right)$ equ. $\wedge x_{1} \ldots \wedge x_{n}\left(\left(\lambda o_{1} \ldots o_{n} \pi\left[o_{1}, \ldots, o_{n}\right], x_{1}, \ldots, x_{n}\right)=c\right)$. 


\section{Sets as Intensional Entities}

Intensional ontology has been developed only as far as it is necessary for my particular semantic purpose. I will not go into applications, and axiomatic or expressive extensions of the system - with the exception of showing how sets (of individuals) can be represented by properties (of individuals).

DEFINITION 24. $S(\tau):=Z^{\langle 0\rangle}(\tau)$ a. $\wedge \mathrm{x}((\tau, \mathrm{x})=\mathrm{t}$ o. $(\tau, \mathrm{x})=\mathrm{c})$; “ $\tau$ is a set iff $\tau$ is a property such that its saturation by any entity is the tautological or contradictory proposition".

DEFINITION 25. $\tau \in \tau^{\prime}:=S\left(\tau^{\prime}\right) a . \tau^{\prime}(\tau)$; " $\tau$ is an element of $\tau^{\prime}$ iff $\tau^{\prime}$ is a set exemplified by $\tau^{\prime \prime}$. Then I can prove what justifies the readings of the above definitions:

The Principle of Comprehension: $\wedge x \vee y(S(y) a . \wedge z(z \in y$ equ. $x(z))$. The Principle of Extensionality: $\wedge x \wedge y(S(x) a . S(y) a$.

$\wedge z(z \in x$ equ. $z \in y)$ imp. $x=y)$.

The Principle of Comprehension is a consequence of the following provable principle:

THEOREM 29. $\wedge z\left(A[z] \operatorname{imp} . Z^{0}(z)\right)$ imp. $\wedge z\left(\lambda o c x^{\prime}\right.$ (non $A[o] a$. $\left.x^{\prime}=c\right)(z)$ equ. $\left.A[z]\right)$. This is how the Principle of Comprehension follows from Theorem 29: let $A[z]$ be $x(z)$, hence $A[o] x(o)$; then $\wedge z\left(x(z)\right.$ imp. $\left.Z^{0}(z)\right)$ by Theorem 20 , and Theorem 29 can be applied; in addition $S\left(\lambda o c x^{\prime}\right.$ (non $\left.A[o] a . x^{\prime}=c\right)$ ) [hence $S\left(\lambda o c x^{\prime}\left(x(o) a . x^{\prime}=\right.\right.$ $c)$ )]: if non $Z^{0}(z)$, then $\left(\lambda o c x^{\prime}\right.$ (non $\left.\left.A[o] a . x^{\prime}=c\right), z\right)=c$ by Axiom 13; if $Z^{0}(z)$ and $A[z]$, then $c x^{\prime}\left(\right.$ non $\left.A[z] a \cdot x^{\prime}=c\right)=t$, hence by Axiom $15\left(\lambda o c x^{\prime}\left(\right.\right.$ non $\left.\left.A[o] a . x^{\prime}=c\right), z\right)=t$; if $Z^{0}(z)$ and non $A[z]$, then $c x^{\prime}$ (non $\left.A[z] a \cdot x^{\prime}=c\right)=c$, hence by Axiom $15\left(\lambda o c x^{\prime}\left(\right.\right.$ non $A[o] a . x^{\prime}=$ $c), z)=c$; hence $\wedge z\left(\left(\lambda o c x^{\prime}\left(\right.\right.\right.$ non $\left.\left.A[o] a . x^{\prime}=c\right), z\right)=t o .\left(\lambda o c x^{\prime}\right.$ (non $\left.\left.\left.A[o] a . x^{\prime}=c\right), z\right)=c\right)$; moreover $Z^{(0)}\left(\lambda o c x^{\prime}\left(\right.\right.$ non $\left.\left.A[o] a . x^{\prime}=c\right)\right)$ by Theorem 27; the rest is obvious.

Theorem 29 states that any monadic predicate applying only to individuals has a set corresponding to it whose elements are precisely the 
individuals the predicate is true of. Its proof: assume (1) $\wedge z(A[z] \mathrm{imp}$. $\left.Z^{0}(z)\right)$;

(i) assume $A[z]$, hence $c x^{\prime}$ (non $A[z] a \cdot x^{\prime}=c$ ) $=t$, hence, since Axiom 15 is applicable $\left[Z^{0}(z)\right.$ by $\left.(1)\right]$ and $T(t)$, by Definition $21 \lambda o c x^{\prime}$ (non $\left.A[o] a \cdot x^{\prime}=c\right),(z)$

(ii) assume non $A[z]$, hence $\left(\lambda o c x^{\prime}\left(\right.\right.$ non $\left.\left.A[o] a . x^{\prime}=c\right), z\right)=c$, no matter whether $Z^{0}(z)$ or non $Z^{0}(z)$ (see above); and non $T(c)$; hence by Definition 21 non $\lambda o c x^{\prime}$ (non $\left.A[o] a . x^{\prime}=c\right)(z)$. (Because of the initial condition $\wedge z\left(A[z]\right.$ imp. $\left.Z^{0}(z)\right)$ Russell's Paradox cannot be derived from Theorem 29; it merely serves to prove the negation of $\wedge z\left(z \notin z\right.$ imp. $\left.Z^{0}(z)\right)$ - which also can be proved independently.)

There is an equally appropriate reading of $S(\tau)$ beside " $\tau$ is a set", namely " $\tau$ is an essential property", since $\left(\tau, \tau^{\prime}\right)=t$ is equivalent to $\wedge y\left(M C(y)\right.$ imp. $(\tau$ in $\left.y)\left(\tau^{\prime}\right)\right)$ (" $\tau$ applies to $\tau^{\prime}$ in every possible world"), and $\left(\tau, \tau^{\prime}\right)=c$ is equivalent to non $\vee y(M C(y) a$. ( $\tau$ in $y)\left(\tau^{\prime}\right)$ ) (" $\tau$ applies to $\tau^{\prime}$ in no possible world"). That sets can be represented by essential properties - of all things! - is a mildly ironic commentary on Quine's militant extensionalism embodied primarily in his well-known polemic against both intensional entities and essentialism.

(C) I am now ready to state a new semantics for first-order modal predicate-logic. I will not describe in detail the object language $L$. Let is suffice that in it free variables are syntactically distinguished from bound variables and called "individual-constants"; its sentential connectives are - (negation), \& (conjunction) and $M$ (possibility); its quantifier is ( $v$ ) ("for all $v$ "); empty occurrences of the quantifier are avoided; if we go from a sentence $s[a]-a$ being an individual-constant - to $(v) s[v]$, then $v$ is a variable not occurring in $s[a]$. The metalanguage is English enriched by the technical expressions of intensional ontology, and of logical syntax and semantics. In the semantics I have in particular int $|\alpha|$ : "the intension of $\alpha$ ", int ${ }_{a}|\alpha|$ : "the intension of $\alpha$, when $a$ is taken out of $\alpha$ ", $s$ : "the property (of individuals) of being spoken of in $L$ ". Expressions are considered to be abstract individuals, and to be self-referring if used in the meta-language. 
1. The Axioms of the Semantics of $L$

AXIOM S1. For every individual-constant $a$ of $L$ : int $|a| \in s$; "the intension of each individual-constant of $L$ is an individual that is an element of the property (the set, the essential property) of being spoken of in $L$ ". Since I assume that there are individual-constants of $L$ (indeed infinitely many), I can deduce from Axiom S1 that $s$ applies to some individual (is not empty). Since $s$ is an essential property, the same (possible) individuals are spoken of in $L$ in each possible world.

AXIOM S2. For every $n$-place predicate-constant $p$ of $L: Z^{\langle n\rangle}$ (int $\left.|p|\right)$; "the intension of each $n$-place predicate-constant of $L$ is an $n$-place relation (between/of individuals)"; 1-place relations are properties.

AXIOM S3. For every $n$-place predicate-constant $p$ of $L$ and all individual-constants $a_{1}, \ldots, a_{n}$ of $L$ : int $\left|p\left(a_{1}, \ldots, a_{n}\right)\right|=($ int $|p|$, in$\mathrm{t}\left|a_{1}\right|, \ldots$, int $\left.\left|a_{n}\right|\right)$; "the intension of an atomic sentence of $L$ is the proposition which is the saturation of the intension of its predicate by the ordered intensions of its individual-constants".

AXIOM S4. For all sentences $s, s^{\prime}$ of $L: \operatorname{int}\left|\left(s \& s^{\prime}\right)\right|=\left(\operatorname{int}|s| \wedge\right.$ int $\left.\left|s^{\prime}\right|\right)$; "the intension of the conjunction of sentences $s$ and $s$ ' of $L$ is the proposition which is the conjunction of the intension of $s$ and the intension of $s^{\prime \prime}$.

AXIOM S5. For all sentences $s$ of $L$ : int $|-s|=\neg$ int $|s|$; "the intension of the negation of a sentence $s$ of $L$ is the proposition which is the negation of the intension of $s$ ".

AXIOM S6. For all sentences $s$ of $L$ : int $|M s|=p_{1}$ (int $\left.|s|\right)$; "the intension of the 'possibilization' of a sentence $s$ of $L$ is the proposition which is the 1-possibility of the intension of $s$ "; $p_{1}(\tau)=d x \vee y\left(x R_{1} y a\right.$. $T(\tau, y)$ ) (compare Definition 20).

AXIOM S7. $\wedge x \wedge y\left(x R_{1} y\right.$ imp. $\left.M C(x) a . M C(y)\right), \wedge x(M C(x)$ imp. $\left.x R_{1} x\right), \wedge x \wedge y\left(x R_{1} y\right.$ imp. $\left.y R_{1} x\right), \wedge x \wedge y \wedge z\left(x R_{1} y\right.$ a. $y R_{1} z$ imp. $\left.x R_{1} z\right)$. Axiom S7 states that $p_{1}$ is an S5-possibility, that is: for all propositions $x: x \rightarrow p_{1}(x), x \rightarrow \neg p_{1}\left(\neg p_{1}(x)\right), p_{1}\left(p_{1}(x)\right) \rightarrow p_{1}(x)$. 
AXIOM S8. For all sentences $(v) g[v]$ of $L$, individual-constants $a$ of $L$ not occurring in $g[v]$, variables $v$ of $L$ not occurring in $g[a]$ : $\operatorname{int}|(v) g[v]|=a^{s}$ (int $\left.{ }_{a}|g[a]|\right)$; "the intension of a universal sentence of $L$ is the $s$-relative all-proposition of the intension of one of its precise instances, when the instantiating individual-constant is taken out of it".

AXIOM S9. For all sentences $s[a]$ of $L$ and individual-constants $a$ of $L$ not in $\lambda o \pi[o]:$ int $|s[a]|=\pi[$ int $|a|]$ imp. int $t_{a}|s[a]|=\lambda o \pi[o]$; "if the intension of a sentence $s[a]$ of $L$ has been analyzed so far that every occurrence of the individual constant $a$ in the analyzing expression " $\pi$ [int $|a|]$ " is preceded by "int|", then the intension of $s[a]$, when $a$ is taken out of it, is the property resulting by the extraction of int $|a|$ from $\pi[$ int $|a|] "$.

\section{Some Important Semantic Concepts}

DEFINITION S1. $s$ is a true sentence of $L:=s$ is a sentence of $L a$. $T($ int $|s|)$.

DEFINITION S2. $s$ is a true sentence of $L$ in $\tau:=s$ is a sentence of L a. $T$ (int $|s|, \tau)$.

DEFINITION S3. $s$ is an ontologically true sentence of $L:=s$ is a sentence of $L a$. int $|s|=t$.

It can be proved:

THEOREM S1. $\wedge x(x$ is an ontologically true sentence of $L$ equ. $\wedge y(M C(y)$ imp. $x$ is a true sentence of $L$ in $y)$.

DEFINITION S4. $s_{1}, \ldots, s_{n} L$-entails $s:=s_{1}, \ldots, s_{n}$ and $s$ are sentences of $L a$. (int $\left|s_{1}\right| \wedge \ldots \wedge$ int $\left.\left|s_{n}\right|\right) \rightarrow$ int $|s| ; \quad$ (equivalently: ...a. (int $\left|s_{1}\right| \wedge \ldots \wedge$ int $\left|s_{n}\right| \wedge \neg$ int $\left.|s|\right)=c$ ).

Being an ontologically true sentence of $L$ is not yet being a logically true sentence of $L$, and $L$-entailment is not yet logical $L$-entailment. The latter concepts will be defined with respect to logical form. In preparation of the definition I need to introduce two notions: 
(a) the complete analysis of a sentence $s$ of $L: A_{s}$;

(b) the all-trivialization of the complete analysis of a sentence $s$ of $L$ : $T_{s}$.

Let $g$ be a sentence of $L$ :

(a) The complete analysis of $g: A_{g}$ is a singular term $\tau$ of the metalanguage such that int $|g|=\tau$ is provable on the basis of Axiom S1-S9, and "int|" occurs in $\tau$ precisely in front of the occurrences of the predicate-constants of $g$ in $\tau$ and precisely in front of the occurrences of the individual-constants of $g$ in $\tau$ (if there are any). (The complete analysis of $g$ is uniquely determined - the choice of extraction-variables $\left(o, o^{\prime}, \ldots\right)$ being inessential.) Here are some complete analyses of sentences of $L$ : the complete analysis of $P_{1}^{2}\left(a, a^{\prime}\right)$ : (int $\left|P_{1}^{2}\right|$, int $|a|$, int $\left.\left|a^{\prime}\right|\right)$; the complete analysis of $(u)\left(u^{\prime}\right) P_{1}^{2}\left(u, u^{\prime}\right)$ :

$$
a^{s}\left(\lambda o^{\prime} \alpha^{s}\left(\lambda o\left(\operatorname{int}\left|P_{1}^{2}\right|, o^{\prime}, o\right)\right)\right)
$$

the complete analysis of $(u)\left(P_{1}^{1}(u) \& P_{2}^{1}(u)\right)$ :

$$
a^{s}\left(\lambda o\left(\left(\operatorname{int}\left|P_{1}^{1}\right|, o\right) \wedge\left(\operatorname{int}\left|P_{2}^{1}\right|, o\right)\right)\right)
$$

the complete analysis of $-(u) P_{1}^{2}\left(u, a^{\prime}\right)$ :

$$
\neg a^{s}\left(\lambda o\left(\operatorname{int}\left|P_{1}^{2}\right|, o, \operatorname{int}\left|a^{\prime}\right|\right)\right) ;
$$

the complete analysis of $-\left((u) P_{1}^{2}\left(u, a^{\prime}\right) \&-P_{1}^{2}\left(a, a^{\prime}\right)\right)$ :

$$
\neg\left(a^{s}\left(\lambda o\left(\operatorname{int}\left|P_{1}^{2}\right|, o, \operatorname{int}\left|a^{\prime}\right|\right)\right) \wedge \neg\left(\operatorname{int}\left|P_{1}^{2}\right|, \operatorname{int}|a|, \text { int }\left|a^{\prime}\right|\right)\right) .
$$

(b) The all-trivialization of the complete analysis of $g: T_{g}$ is a sentence $\sigma$ of the meta-language constructed from $A_{g}$ as follows:

First step: write down $A_{g}$ and add " $=t$ ".

Second step: replace any expression of the form int $|\alpha|$ in $A_{g}$, where $\alpha$ is an individual-constant, everywhere in $A_{g}$ by a normal variable of the metalanguage (different variables for different expressions); the expression obtained is $A_{g}\left[x_{1}, \ldots, x_{n}\right]=t$ (where $x_{1}, \ldots, x_{n}$ represent the variables used); then go to 
$\wedge x_{1} \ldots \wedge x_{n}\left(x_{1} \in s a . \ldots a . x_{n} \in s\right.$ imp. $\left.A_{g}\left[x_{1}, \ldots, x_{n}\right]=t\right)$

(the ordering and typographical appearance of the variables is inessential).

Third step: replace any expression of the form int $|\alpha|$ in $A_{g}$, where $\alpha$ is a predicate-constant, everywhere in $A\left[x_{1}, \ldots, x_{n}\right]$ by a normal variable of the meta-language (different variables for different expressions, the variables used must not occur in the expression obtained in the second step); the expression obtained is $\wedge x_{1} \ldots \wedge x_{n}\left(x_{1} \in s a . \ldots a . x_{n} \in s\right.$ imp. $A_{g}\left[x_{1}, \ldots, x_{n} ; y_{1}, \ldots, y_{k}\right]=t$ ) (where $y_{1}, \ldots, y_{k}$ represent the variables used); at the same time write down $Z^{\langle m(r)\rangle}\left(y_{r}\right)$ - where $m(r)$ is the number of places of the predicate-constant $\alpha_{r}$ such that int $\left|\alpha_{r}\right|$ is replaced by $y_{r} ;$ then go to $\wedge y_{1} \ldots \wedge y_{k}\left(Z^{\langle m(1)\rangle}\left(y_{1}\right) a . \ldots a . Z^{\langle m(k)\rangle}\left(y_{k}\right)\right.$ imp. $\wedge x_{1} \ldots \wedge x_{n}\left(x_{1} \in s \quad a . \ldots a . \quad x_{n} \in s\right.$ imp. $A_{g}\left[x_{1}, \ldots, x_{n}\right.$; $\left.\left.\left.y_{1}, \ldots, y_{k}\right]=t\right)\right)$.

Fourth step: replace $s$ in the expression obtained in the third step everywhere by a variable $z$ not yet used and go to $\wedge z[\vee y(y \in z)$ imp. $\wedge y_{1} \ldots \wedge y_{k}\left(Z^{\langle m(1)\rangle}\left(y_{1}\right) \quad\right.$ a. ...a. $Z^{\langle m(k)\rangle}\left(y_{k}\right) \quad$ imp.

$\wedge x_{1} \ldots \wedge x_{n}\left(x_{1} \in z\right.$ a. . . a. $x_{n} \in z$ imp. $A_{g}\left[x_{1}, \ldots, x_{n} ; y_{1}, \ldots, y_{k}\right.$; $z]=t))$ ]. Here are two examples of the all-trivializations of complete analyses of sentences of $L$ :

the all-trivialization of the complete analysis of $P_{1}^{2}\left(a, a^{\prime}\right)$ : $\wedge z\left[\vee y(y \in z)\right.$ imp. $\wedge y^{\prime}\left(Z^{\langle 2\rangle}\left(y^{\prime}\right)\right.$ imp. $\wedge x \wedge x^{\prime}(x \in z a$. $x^{\prime} \in z$ imp. $\left.\left.\left.\left(y^{\prime}, x, x^{\prime}\right)=t\right)\right)\right]$, in short: $\wedge z \wedge y^{\prime} \wedge x \wedge x^{\prime}$ $\left(\vee y(y \in z) a . Z^{\langle 2\rangle}\left(y^{\prime}\right) a . x \in z a \cdot x^{\prime} \in z\right.$ imp. $\left.\left(y^{\prime}, x, x^{\prime}\right)=t\right)$;

the all-trivialization of the complete analysis of $-\left((u) P_{1}^{2}\left(u, a^{\prime}\right) \quad \& \quad-P_{1}^{2}\left(a, a^{\prime}\right)\right): \wedge z[\vee y(y \in z) \quad$ imp. $\wedge y^{\prime}\left(Z^{\langle 2\rangle}\left(y^{\prime}\right)\right.$ imp. $\wedge x \wedge x^{\prime}\left(x \in z \quad\right.$ a. $\quad x^{\prime} \in z \quad$ imp. $\left.\left.\left.\neg\left(a^{z}\left(\lambda o\left(y^{\prime}, o, x^{\prime}\right)\right) \wedge \neg\left(y^{\prime}, x, x^{\prime}\right)\right)=t\right)\right)\right]$, in short: $\wedge z \wedge y^{\prime}$ $\wedge x \wedge x^{\prime}\left(\vee y(y \in z)\right.$ a. $Z^{\langle 2\rangle}\left(y^{\prime}\right)$ a. $x \in z$ a. $x^{\prime} \in z$ imp. $\left.\neg\left(a^{2}\left(\lambda o\left(y^{\prime}, o, x^{\prime}\right)\right) \wedge \neg\left(y^{\prime}, x, x^{\prime}\right)\right)=t\right)$.

Now:

DEFINITION S5. $s$ is a logically true sentence of $L:=s$ is a sentence of $L a . T_{s}$.

DEFINITION S6. $s_{1}, \ldots, s_{n}$ logically $L$-entails $s:=-\left(s_{1} \& \ldots \& s_{n}\right.$ $\&-s)$ is a logically true sentence of $L$. 
According to Definition S5 $P_{1}^{2}\left(a, a^{\prime}\right)$ is not a logically true sentence of $L: a$ and $a^{\prime}$ are two different individuals (remember that expressions are considered to be abstract individuals); consider $\lambda o c x$ (non $(o=a o$. $\left.o=a^{\prime}\right) a . x=c$ ), in short: $\left\{a, a^{\prime}\right\}$; this property is a set; hence we have by Theorem 29, Definition $25 a \in\left\{a, a^{\prime}\right\}, a^{\prime} \epsilon\left\{a, a^{\prime}\right\}, \bigvee y\left(y \in\left\{a, a^{\prime}\right\}\right)$; consider moreover $\lambda o o^{\prime} c x$ (non $o=o^{\prime} a . x=c$ ), in short: id ("identity (between individuals)"); we have $Z^{\langle 2\rangle}(i d)$ and (id, $\left.a, a^{\prime}\right)=c$ (since $a$ and $a^{\prime}$ are different), hence non $\left(i d, a, a^{\prime}\right)=t$; this falsifies the alltrivialization of the complete analysis of $P_{1}^{2}\left(a, a^{\prime}\right)$. The all-trivialization of the complete analysis of $-\left((u) P_{1}^{2}\left(u, a^{\prime}\right) \&-P_{1}^{2}\left(a, a^{\prime}\right)\right)$ is, however, provably true; this makes this sentence of $L$ a logically true sentence of $L$, which means according to Definition S6 that $(u) P_{1}^{2}\left(u, a^{\prime}\right)$ logically $L$-entails $P_{1}^{2}\left(a, a^{\prime}\right)$ - as is desired.

\section{Some Results and Problems}

It is provable:

The Law of Generalization: If $s[a]$ is a logically true sentence of $L$ and the individual-constant $a$ does not occur in $(v) s[v]$ ( $v$ a variable of $L$ not in $s[a])$, then $(v) s[v]$ is a logically true sentence of $L$.

The Law of Necessitation: If $s$ is a logically true sentence of $L$, then $-M-s$ is a logically true sentence of $L$.

I give the proof of the latter law (which is shorter and simpler than the proof of the former): assume $s$ is a logically true sentence of $L$; hence $T_{s}$; we need to show $T_{-M-s} ; T_{s}$ has the shape -.-(..imp. $\left.A_{s}[\#]=t\right)$, $T_{-M-s}$ has the shape ---(...imp. $\left.A_{-M-s}[\#]=t\right)$ (in both schemata -.-, $\ldots$ and \# stand for the very same sequence of expressions), where $A_{-M-s}[\#]$ has the shape $\neg p_{1}\left(\neg A_{s}[\#]\right)$; hence we need to show -.$\left(\ldots \neg p_{1}\left(\neg A_{s}[\#]\right)=t\right)$; now: $\wedge x\left(x=t\right.$ imp. $\left.\neg p_{1}(\neg x)=t\right)$, since $\neg p_{1}(\neg t)=t$, since $\neg d x \vee y\left(x R_{1} y\right.$ a. $\left.T(\neg t, y)\right)=t$, since non $\vee x$ $\vee y\left(x R_{1} y\right.$ a. $\left.T(\neg t, y)\right)$ [non $\bigvee x\left(Z^{1}(x) a . A[x]\right)$ imp. $d x A[x]=c, \neg c=$ $t$, since there is no possible world in which $\neg t(=c)$ is true; this establishes: what we needed to show $-T_{-M-s}$ - follows from $T_{s}$.

There is no difficulty in describing an axiom-system $M P L$ in $L$ which is appropriate for the semantics of $L$. Intensional ontology, even in the elementary state presented in this paper, allows to define the concept 
of a finite sequence (of individuals), which is necessary for the definition of $M P L$-provability:

DEFINITION 26. $\tau$ is a $n$-place
$\vee x_{1} \ldots \vee x_{n}\left(\left(\tau, x_{1}, \ldots, x_{n}\right)=t\right.$ sequence : $=$
o. ..o. non $\left.\left.y_{n}=x_{n} \operatorname{imp} .\left(\tau, y_{1}, \ldots, y_{n}\right)=c\right)\right)$.

DEFINITION 27. $\left\langle\tau_{1}, \ldots, \tau_{n}\right\rangle:=\lambda o_{1} \ldots o_{n} c x\left(\left(\right.\right.$ non $o_{1}=\tau_{1} o \ldots . o$. non $\left.\left.o_{n}=\tau_{n}\right) a . x=c\right) ;\left\langle\tau_{1}, \ldots, \tau_{n}\right\rangle$ is an $n$-place sequence, if (and only if) $\tau_{1}, \ldots, \tau_{n}$ are individuals; for then we have $\left(\left\langle\tau_{1}, \ldots, \tau_{n}\right\rangle, \tau_{1}, \ldots, \tau_{n}\right)=t$ and $\wedge y_{1} \ldots \wedge y_{n}$ (non $y_{1}=\tau_{1} o \ldots o$. non $y_{n}=\tau_{n}$ imp. $\left.\left(\left\langle\tau_{1}, \ldots, \tau_{n}\right\rangle, y_{1}, \ldots, y_{n}\right)=c\right)$; it is easy to prove the accustomed necessary identity condition for $n$-place sequences $\left\langle\tau_{1}, \ldots, \tau_{n}\right\rangle$ and $\left\langle\rho_{1}, \ldots, \rho_{n}\right\rangle$.

DEFINITION 28. $\tau$ is a finite sequence $:=\bigvee \sigma(\tau$ is a $\sigma$-place sequence). Natural numbers are not presupposed for this definition; the existential quantification is substitutional: " $\vee \sigma(\tau$ is a $\sigma$-place sequence)" simply means: for some appropriate substitution for $\sigma$ - an arabic numeral - " $\tau$ is a $\sigma$-place sequence" turns into a true sentence.

It will present no problem to prove the semantic soundness of $M P L$; the semantic completeness of $M P L$ is a different matter (provided, of course, that the background theory is not strengthened to such an extent as to become equal in power to the set-theory which is necessary for constructing a Henkin-Proof, and provided that the given semantical definitions are used).

Another open problem is how definite descriptions, if introduced into $L$, are to be treated semantically. Such singular terms can be considered in two different manners: The reference of definite descriptions is allowed to vary from one possible world to another; or the reference of definite descriptions is not allowed to vary from one possible world to another, but stays fixed to the real-world referent. ${ }^{7}$ In the latter case definite descriptions can be treated in my semantics as it stands:

AXIOM S10. For all singular terms $\iota v g[v]$ of $L$, individual-constants $a$ of $L$ not occurring in $g[v]$, variables $v$ of $L$ not occurring in $g[a]:$ [ $\vee$ ! $z(z \in s a$. int $|g[a]|(z))$ imp. int $|\iota v g[v]|=\iota z(z \in s a$. int $|g[a]|(z))] a$. 
[non $\vee ! z(z \in s$ a. int $|g[a]|(z))$ imp. int $|\iota v g[v]|=$ int $\left.\left|a^{\prime \prime}\right|\right]$. ( $\vee !$ : there is precisely one).

In the former case a contextual definition of the description operator seems to be the most promising approach (this would be in consistency with the Russellian bend of this semantic theory).

The standard practice of defining interpretations of modalized languages can be paralleled more closely if I introduce in general properties and relations of propositions, and in particular $m$ : the property of being a possible world considered in $L$, and $r$ : the accessibility-relation between possible worlds considered in $L$. The background theory, the semantic axioms and the definition of the complete analysis and the all-trivialization of the complete analysis of a sentence of $L$ have to be changed accordingly. The logic of the possibility-operator $M$ of $L$ thereby becomes the logic of no particular possibility-operator, but that of a whole set of such operators. (Analogously the logic of the all-quantifier of $L$ is already the logic of no particular all-quantifier, but that of a whole set of such quantifiers.)

\section{NOTES}

1 A theory of basic intensional entities has been developed by G. Bealer in Quality and Concept. Mine is rather different from his. The basic concepts are different, and while Bealer's theory in its fully developed state resembles axiomatic set-theory, mine resembles type-theory, more accurately the so-called standard theory of types, where type-distinctions are expressed by predicates, and not incorporated into the syntax of the language. Both theories, however, are first-order theories, and both regard sets - in an analogous manner - as special properties.

2 On the whole there are two set-theoretic ontologies of intensional entities. According to the classical approach based on R. Carnap's Meaning and Necessity (and more remotely on Frege) possible worlds are entities sui generis, and properties (for example) are functions (i.e., special sets) that assign to each possible world and possible individual (not world-bound) a truth-value ( $\emptyset$ or $\{\emptyset\}$ ), propositions functions that assign to each possible world a truth-value. According to $\mathrm{D}$. Lewis, on the other hand, properties are sets of (world-bound) possible individuals, propositions sets of possible worlds, which are for him possible individuals (hence propositions are properties!); see for example $O n$ the Plurality of Worlds, p. 50, p. 53.

${ }^{3}$ My basic semantic paradigm is that of Russell, not that of Frege. (For an exposition and defense of Russell's theory of meaning see Quality and Concept, p. 160ff.) But there is the following difference to Russell. According to Russell, sentences (for example) mean propositions (in his sense). I transform Russell's theory of meaning into a theory of intension and say accordingly: sentences intend - as part of their meaning - propositions 
(in my sense). 1 do not aim in this paper at developing a theory of meaning. The Russellian paradigm favors an algebraic treatment of semantics. This will be the treatment I accord to the semantics of $L$. It is much simpler and more economic in basic concepts than Bealer's 'New Semantic Method' in Quality and Concept, Section 13 and 14 of Chapter 2.

${ }^{4}$ For this concept and the concept (complete) atomistic Boolean algebra compare Tarski's classical paper 'On the Foundations of Boolean Algebra', p. 321ff, p. 335. If Tarski's system $\mathscr{B}_{1}-\mathscr{B}_{4}$ is rewritten in the language of intensional ontology here employed (settheoretical expressions have to be represented (approximated) by non-set-theoretical means) and is applied to propositions, it turns out to be (deductively) equivalent to Axioms $1-5$, Axiom 0 being presupposed. Tarski's system $\mathscr{B}_{1}-\mathscr{B}_{4}, \mathscr{D}-$ having been subjected to the same treatment - turns out to be equivalent to Axioms $1-6$, Axiom 0 being presupposed. ( $\mathscr{D}$ is the axiom of atomism; Tarski's definition of an atom is different from mine!)

5 This conception of possible worlds is anticipated by A. Plantinga in The Nature of Necessity, p. 45: ". . . a possible world is simply a possible state of affairs that is maximal". If this is intended to be a definition, then the concept of a possible world cannot be used for defining the concept of possibility. But this defect is easily remedied: simply replace "possible" in "possible state of affairs" by "consistent".

The relation of Axioms 0-9 to the standard approach which considers (coarse-grained) propositions to be sets of possible worlds is as follows: Let $\mathrm{PW}$ be the set of possible worlds; $Z^{\prime}(\tau):=\tau \subset P W ; \tau P \tau^{\prime}:=\tau \subset P W a . \tau^{\prime} \subset P W a . \tau^{\prime} \subset \tau$. Given these definitions, which model intensional relations by extensional ones, the Axioms 0-6 are easily verified within the framework of set-theory, and we have the following provable correspondences:

$$
\begin{aligned}
& c x A[x]=\cap\left\{Z^{1}(x): A[x]\right\}, \text { if }\left\{Z^{1}(x): A[x]\right\} \text { is not empty; else } \\
& c x A[x]=P W ; \\
& d x A[x]=\cup\left\{Z^{1}(x): A[x]\right\} ; \\
& \wedge x(M i(x) \text { equ. } x=P W), \wedge x(A t(x) \text { equ. } x=P W \text { o. } \vee y(y \in P W a . x= \\
& P W-\{y\})), t=P W, c=\emptyset, \wedge x(M a(x) \text { equ. } x=\emptyset), \wedge x(\text { To(x) equ. } x=\emptyset \\
& \text { o. } \vee y(y \in P W \text { a. } x=\{y\})), \wedge x(M C(x) \text { equ. } \vee y(y \in P W a . x=\{y\})) .
\end{aligned}
$$

The axioms 7-9 amount to stating $\vee y(y \in P W a . w=\{y\}) a$. non $w=P W$.

${ }^{6} \mathrm{G}$. Bealer regards the possible-worlds definition of necessity as circular, since the concept necessarily false proposition needs to be employed in the definition of possible world. He defines (Quality and Concept, p. 206):

$w$ is a world : = for every proposition $x, x$ is in $w$ or the negation of $x$ is in $w$ (in my terms: $w$ is a maximal proposition); $w$ is a possible world $:=w$ is a world and no necessarily false proposition is-true-in $w$ (in my terms: $w$ is a maximal and consistent proposition).

There is, of course, no need to employ the concept of a necessarily false proposition in the latter definition, as I have shown in this paper.

${ }^{7}$ Kaplan's dthat forms constant definite descriptions out of variables ones. Both uses are based on ordinary language. 


\section{REFERENCES}

Bealer, G.: 1983, Quality and Concept, Clarendon Press, Oxford.

Carnap, R.: 1967, Meaning and Necessity, The University of Chicago Press, Chicago.

Kaplan, D.: 1979, 'Dthat', in French, Uehling and Wettstein (eds.), Contemporary

Perspectives in the Philosophy of Language, University of Minnesota Press, pp. 6-27.

Lewis, D.: 1986, On the Plurality of Worlds, Basil Blackwell, Oxford.

Meixner, U.: 1991, Axiomatische Ontologie, Roderer Verlag, Regensburg.

Plantinga, A.: 1974, The Nature of Necessity, Clarendon Press, Oxford.

Tarski, A.: 1956, 'On the Foundations of Boolean Algebra', in Tarski: Logic, Semantics, Metamathematics, Clarendon Press, Oxford, pp. 320-341.

Manuscript submitted 23 January, 1991

Final version received 28 October, 1991

Dept. of Philosophy

University of Regensburg

Universitätstrasse 31

8400 Regensburg

Germany 